\title{
A Woman's Job, A Man's Privilege? Identifying Ethical, Racial, Societal Factors in the Prevailing Gender Disparity in Organ Donation
}

Tanishqa Sheth ${ }^{1}$, Rahul Rangan ${ }^{2}$, Alisha Jain $^{3}$, Pranjal Vatnani ${ }^{4}$, Aman Saxena ${ }^{5}$, Jhancy Rani $^{6}$, Shiv H. Joshi ${ }^{7}$

${ }^{1}$ Undergraduate student, K.J. Somaiya Medical College, Mumbai,

${ }^{2}$ Undergraduate student, Krishna Institute of Medical Sciences, Karad,

${ }^{3}$ Undergraduate student, K.J. Somaiya Medical College, Mumbai

${ }^{4}$ Undergraduate student, K.J. Somaiya Medical College, Mumbai

${ }^{5}$ Undergraduate student, D.Y. Patil University- School of Medicine, Navi Mumbai

${ }^{6}$ Undergraduate student, Velammal Medical College and Research Institute, Madurai

${ }^{7}$ Head Student Bioethics Program (India) of the International Chair in Bioethics.

Corresponding Author: Shiv H. Joshi

E-mail:drshivjoshi93@gmail.com

\begin{abstract}
Background: Women have been major organ donors in the world while men have predominantly played the role of recipients. This, along with various socio-cultural, racial, and economic factors as well as personal attitudes and beliefs, has made it difficult to meet the growing demand of organs for transplantation. The aim of the study was to investigate the prevailing degree of gender disparity in organ donation and to find out factors which contribute to such disparity.

Methodology: Search were performed using the MeSH terms and the related keywords on MEDLINE. A total of 47 out of 60 articles were included. This study analyzed the various reviews on the association between gender and organ transplantation, and the factors that may influence the same.

Results: We found that there exists a gender disparity not only where women donate more but men are the major recipients, and that multiple other factors medical or otherwise either directly or indirectly contribute to the same. Despite males and females having equal likelihood of getting through the required steps to be approved as a donor, females have shown more likelihood to agree to donate their organs, live or otherwise. Men are more likely to donate for their children than for their wives. Male and younger recipients are favored, while females and middle-aged or older donors are preferred.

Conclusion: Gender disparity in organ donation exists throughout the world and there is a need to bridge this gender gap through a concerted and cohesive plan of action.
\end{abstract}

Keywords: Gender, Bioethics, Transplantation, Organ Donation, Women Health

\section{Introduction}

A larger number of live organ transplantations are facilitated by women as donors than men [1]. Out of all transplants, $54.7 \%$ women were donors. A striking contrast was observed with only $39.4 \%$ of women being recipients [2]. In such progressive times, organs that are crucial for survival remain inequitable. Many studies conducted all around the world have established this trend. In cases of liver transplants women are more likely to die on the waitlist [3]. In the ever-evolving field 
of transplant medicine, there exists a gap and to make organ transplantation accessible to all, this gender gap must be bridged. Benevolence does not only pertain to women. Myths must be driven away, and correct education must be imparted to all minority communities, all genders and all age groups to allow the supply of organs to meet the growing demand. This review analyses the injustice women face when transplant is the only treatment option and its by-products like further complications. The education one receives also shows a conspicuous trend [4]. In a study conducted, it was established that women have less knowledge and are less informed about transplant procedures and therapy, and yet have a greater sense of responsibility to undertake the role of a donor [7]. 13.5\% more students in the medical fraternity are willing to donate as opposed to that of students majoring in economics [5]. This review assesses the interplay between the emotional, socioeconomic, racial, educational and ethical aspects in the gender disparity in organ donation.

\section{Research objectives}

- To scope for and establish the prevailing degree of gender disparity in organ donation.

- To identify and discuss what factors contribute to the variance in the degree of disparity, if any.

\section{Review approach}

The research question entailed assessing the relevance of gender in organ transplantation. A search was done by using PubMed to find all original and review articles with original reports, published from the period of January 2000 to February 2020. A combination of keywords with Medical Subject Headings $(\mathrm{MeSH})$ related to gender and organ donation was used. The following $\mathrm{MeSH}$ terms were used interchangeably and in combination, so as to find all relevant articles which encompassed disparity in organ donation with respect to gender: 'gender', 'gender disparity', 'organ donation', 'transplant medicine', 'ethics', 'bioethics', 'religion', 'religious beliefs', 'BAME', 'race', 'women', 'donor', 'recipient'.

\section{Inclusion and exclusion criteria}

The articles to be analysed were included if they were found using the aforementioned search process for our review, with respect to the above listed databases, MeSH and search terms. All articles having free full text available through PubMed were included in our review. We included studies which spoke about the importance of gender in organ transplantation and did not limit to a specific organ. Case reports, letters to editors and commentaries were excluded in the scope of this review.

\section{Data Review}

Table 1: Details and decision to include/exclude the retrieved reviews on the association between gender and organ transplantation.

\begin{tabular}{|c|c|c|c|c|c|c|c|c|}
\hline $\begin{array}{l}\text { Sr. } \\
\text { No. }\end{array}$ & $\begin{array}{c}\text { PubMed } \\
\text { Reference } \\
\text { ID }\end{array}$ & Year & $\begin{array}{c}\text { Endpoint } \\
\text { (measurement) }\end{array}$ & $\begin{array}{l}\text { Type of } \\
\text { Study }\end{array}$ & $\begin{array}{c}\text { Inclusion/Exclusion } \\
\text { Criteria }\end{array}$ & $\begin{array}{l}\text { Included } \\
\quad \text { or } \\
\text { excluded }\end{array}$ & $\begin{array}{c}\text { Journal } \\
\text { Published }\end{array}$ & $\begin{array}{l}\text { Article } \\
\text { Source }\end{array}$ \\
\hline 1 & 3999296 & 1985 & $\begin{array}{l}\text { Public attitudes and } \\
\text { behaviour about } \\
\text { organ donation }\end{array}$ & $\begin{array}{l}\text { Original } \\
\text { Article }\end{array}$ & $\begin{array}{l}\text { No inclusion/exclusion } \\
\text { criteria as part of the article. }\end{array}$ & Excluded & JAMA & $\begin{array}{l}\text { National } \\
\text { Library of } \\
\text { Medicine }\end{array}$ \\
\hline 2 & 10977785 & 2000 & $\begin{array}{l}\text { Gender disparity in } \\
\text { living renal } \\
\text { transplant donation }\end{array}$ & $\begin{array}{l}\text { Original } \\
\text { Article }\end{array}$ & $\begin{array}{l}\text { family members of living } \\
\text { donor allograft recipients at } \\
\text { a single large center over a } \\
\text { 5-year period }(\mathrm{n}=144) .\end{array}$ & Included & $\begin{array}{l}\text { American } \\
\text { Journal of } \\
\text { Kidney } \\
\text { Diseases } \\
\end{array}$ & $\begin{array}{l}\text { National } \\
\text { Library of } \\
\text { Medicine }\end{array}$ \\
\hline 3 & 11821739 & 2002 & $\begin{array}{l}\text { Gender imbalance } \\
\text { in living donor } \\
\text { renal } \\
\text { transplantation }\end{array}$ & $\begin{array}{l}\text { Original } \\
\text { Article }\end{array}$ & $\begin{array}{l}\text { Electronic medical records } \\
\text { of all living related and } \\
\text { living unrelated kidney } \\
\text { transplants performed } \\
\text { between } 1964 \text { and } 2000\end{array}$ & Included & $\begin{array}{l}\text { Transplanta } \\
\text { tion }\end{array}$ & $\begin{array}{l}\text { Wolters } \\
\text { Kluwer }\end{array}$ \\
\hline
\end{tabular}




\begin{tabular}{|c|c|c|c|c|c|c|c|c|}
\hline & & & & & were reviewed & & & \\
\hline 4 & 12741404 & 2002 & $\begin{array}{c}\text { Giving until it } \\
\text { hurts: prisoners are } \\
\text { not the answer to } \\
\text { the national organ } \\
\text { shortage }\end{array}$ & $\begin{array}{l}\text { Topic for } \\
\text { Debate }\end{array}$ & $\begin{array}{l}\text { No inclusion/exclusion } \\
\text { criteria as part of the } \\
\text { debate. }\end{array}$ & Excluded & $\begin{array}{l}\text { Indiana } \\
\text { Law } \\
\text { Review }\end{array}$ & $\begin{array}{l}\text { National } \\
\text { Library of } \\
\text { Medicine }\end{array}$ \\
\hline 5 & 15859919 & 2003 & $\begin{array}{l}\text { Ethical issues in } \\
\text { organ and tissue } \\
\text { transplantation }\end{array}$ & $\begin{array}{l}\text { Topic for } \\
\text { Debate }\end{array}$ & $\begin{array}{l}\text { No inclusion/exclusion } \\
\text { criteria as part of the } \\
\text { analysis. }\end{array}$ & Excluded & $\begin{array}{l}\text { Experiment } \\
\text { al and } \\
\text { Clinical } \\
\text { Transplanta } \\
\text { tion }\end{array}$ & $\begin{array}{l}\text { National } \\
\text { Library of } \\
\text { Medicine }\end{array}$ \\
\hline 6 & 15501205 & 2004 & $\begin{array}{l}\text { The net transfer of } \\
\text { transplant organs } \\
\text { across race, sex, } \\
\text { age, and income }\end{array}$ & $\begin{array}{l}\text { Original } \\
\text { Article }\end{array}$ & $\begin{array}{l}\text { Subjects included were all } \\
\text { transplant donors in the } \\
\text { United States from } 1996 \text { to } \\
2001.6 \% \text { of subjects who } \\
\text { were neither black nor } \\
\text { white were excluded. }\end{array}$ & Included & $\begin{array}{l}\text { The } \\
\text { American } \\
\text { Journal of } \\
\text { Medicine }\end{array}$ & Elsevier \\
\hline 7 & 15701672 & 2005 & $\begin{array}{l}\text { Gender imbalance } \\
\text { among donors in } \\
\text { living kidney } \\
\text { transplantation: the } \\
\text { Norwegian } \\
\text { experience }\end{array}$ & $\begin{array}{l}\text { Original } \\
\text { Article }\end{array}$ & $\begin{array}{l}\text { Patients }>18 \text { years were } \\
\text { included and the medical } \\
\text { criteria of : Kidney function } \\
\text { must be normal with } \\
\text { creatinine clearance }>80 \\
\text { ml/min and the urine } \\
\text { specimen must be normal. } \\
\text { Blood pressure should be in } \\
\text { the normotensive range } \\
\text { (<140/90 mmHg). X-ray of } \\
\text { the chest as well as exercise } \\
\text { echocardiogram in donors } \\
>40 \text { years of age must be } \\
\text { normal. Body mass index } \\
>30 \mathrm{~kg} / \mathrm{m} 2 \text { and elevated } \\
\text { fasting blood glucose are } \\
\text { exclusion criteria. }\end{array}$ & Included & $\begin{array}{l}\text { Nephrol } \\
\text { Dialysis } \\
\text { Transplant }\end{array}$ & $\begin{array}{l}\text { Oxford } \\
\text { Academic }\end{array}$ \\
\hline 8 & 16127268 & 2005 & $\begin{array}{c}\text { Kidney } \\
\text { transplantation and } \\
\text { gender disparity }\end{array}$ & $\begin{array}{c}\text { Original } \\
\text { Report }\end{array}$ & $\begin{array}{l}\text { No inclusion/exclusion } \\
\text { criteria as part of the study } \\
\text { analysis. }\end{array}$ & Included & $\begin{array}{l}\text { American } \\
\text { Journal of } \\
\text { Nephrol }\end{array}$ & Karger \\
\hline 9 & 15696526 & 2005 & $\begin{array}{l}\text { Ethics, } \\
\text { transplantation, } \\
\text { and the changing } \\
\text { role of anatomists }\end{array}$ & $\begin{array}{l}\text { Topic for } \\
\text { Debate }\end{array}$ & $\begin{array}{l}\text { No inclusion/exclusion } \\
\text { criteria as part of the } \\
\text { analysis. }\end{array}$ & Excluded & $\begin{array}{c}\text { Clinical } \\
\text { Anatomy }\end{array}$ & $\begin{array}{l}\text { National } \\
\text { Library of } \\
\text { Medicine }\end{array}$ \\
\hline 10 & 16181018 & 2005 & $\begin{array}{l}\text { Gender-specific } \\
\text { issues in liver and } \\
\text { kidney failure and } \\
\text { transplantation: a } \\
\text { review }\end{array}$ & $\begin{array}{c}\text { Review } \\
\text { Article }\end{array}$ & $\begin{array}{l}\text { No inclusion/exclusion } \\
\text { criteria as part of the } \\
\text { review. }\end{array}$ & Included & $\begin{array}{l}\text { Journal of } \\
\text { Women's } \\
\text { Health }\end{array}$ & $\begin{array}{l}\text { National } \\
\text { Library of } \\
\text { Medicine }\end{array}$ \\
\hline 11 & 17164705 & 2006 & $\begin{array}{l}\text { Gender disparities } \\
\text { in the live kidney } \\
\text { donor evaluation } \\
\text { process }\end{array}$ & $\begin{array}{c}\text { Original } \\
\text { Article }\end{array}$ & $\begin{array}{c}\text { Potential donors were } \\
\text { evaluated for live donor } \\
\text { kidney transplantation } \\
\text { between January 1, 2000, } \\
\text { and January 1, 2004, if they } \\
\text { fulfilled the adequate donor } \\
\text { criteria. }\end{array}$ & Included & $\begin{array}{l}\text { Transplanta } \\
\text { tion }\end{array}$ & $\begin{array}{l}\text { Wolters } \\
\text { Kluwer }\end{array}$ \\
\hline 12 & 16857540 & 2006 & $\begin{array}{l}\text { Ethnic and gender } \\
\text { differences in } \\
\text { willingness among } \\
\text { high school } \\
\text { students to donate } \\
\text { organs }\end{array}$ & $\begin{array}{c}\text { Original } \\
\text { Article }\end{array}$ & $\begin{array}{c}883 \text { students attending } \\
\text { health science class at nine } \\
\text { inner-city high schools in } \\
\text { Seattle, Washington }\end{array}$ & Included & $\begin{array}{l}\text { Journal of } \\
\text { Adolesc } \\
\text { Health }\end{array}$ & Elsevier \\
\hline 13 & 18089300 & 2007 & $\begin{array}{l}\text { Gender bias in } \\
\text { renal } \\
\text { transplantation: are } \\
\text { women alone } \\
\text { donating kidneys in } \\
\text { India? }\end{array}$ & $\begin{array}{c}\text { Original } \\
\text { Article }\end{array}$ & $\begin{array}{l}\text { Retrospective analysis of all } \\
\text { LD renal transplantations } \\
\text { performed at a single center } \\
\text { between } 2001 \text { and } 2005\end{array}$ & Included & $\begin{array}{c}\text { Transplant } \\
\text { Proceed }\end{array}$ & Elsevier \\
\hline
\end{tabular}




\begin{tabular}{|c|c|c|c|c|c|c|c|c|}
\hline & & & & & & & & \\
\hline 17 & 18580010 & 2008 & $\begin{array}{l}\text { Gender disparity in } \\
\text { kidney } \\
\text { transplantation }\end{array}$ & $\begin{array}{l}\text { Original } \\
\text { Article }\end{array}$ & $\begin{array}{l}\text { The data of age, gender, } \\
\text { nationality, donor type and } \\
\text { waiting time before } \\
\text { transplantation of } 1426 \\
(61.85 \% \text { male, } 38.14 \% \\
\text { female) recipients who } \\
\text { underwent transplantation } \\
\text { in Imam Reza Hospital in } \\
\text { the northeast of Iran from } \\
1990 \text { to } 2003 \text {, was analysed. }\end{array}$ & Included & $\begin{array}{c}\text { Saudi } \\
\text { Journal of } \\
\text { Kidney } \\
\text { Diseases } \\
\text { and } \\
\text { transplantat } \\
\text { ion }\end{array}$ & $\begin{array}{l}\text { National } \\
\text { Library of } \\
\text { Medicine }\end{array}$ \\
\hline 18 & 18190416 & 2008 & $\begin{array}{l}\text { Ethics of organ } \\
\text { donation and } \\
\text { transplantation } \\
\text { involving prisoners: } \\
\text { the debate extends } \\
\text { beyond our borders }\end{array}$ & $\begin{array}{c}\text { Review } \\
\text { Article }\end{array}$ & $\begin{array}{l}\text { No inclusion/exclusion } \\
\text { criteria as part of the } \\
\text { review. }\end{array}$ & Excluded & $\begin{array}{l}\text { Internal } \\
\text { medicine } \\
\text { journal }\end{array}$ & $\begin{array}{l}\text { Wiley } \\
\text { Library }\end{array}$ \\
\hline 20 & 19469039 & 2009 & $\begin{array}{l}\text { Attitudes, beliefs } \\
\text { and behaviours } \\
\text { surrounding organ } \\
\text { donation among } \\
\text { Hispanic women }\end{array}$ & $\begin{array}{c}\text { Review } \\
\text { Article }\end{array}$ & $\begin{array}{l}\text { No inclusion/exclusion } \\
\text { criteria as part of the } \\
\text { review. }\end{array}$ & Included & $\begin{array}{l}\text { Current } \\
\text { Opin } \\
\text { Organ } \\
\text { Transplant }\end{array}$ & $\begin{array}{l}\text { Wolters } \\
\text { Kluwer }\end{array}$ \\
\hline 21 & 21265292 & 2010 & $\begin{array}{l}\text { Attitudes toward } \\
\text { organ donation and } \\
\text { donor behaviour: a } \\
\text { review of the } \\
\text { international } \\
\text { literature }\end{array}$ & $\begin{array}{l}\text { Review } \\
\text { Article. }\end{array}$ & $\begin{array}{l}\text { members of the public (i.e., } \\
\text { not transplant recipients, } \\
\text { donor families, or health } \\
\text { professionals) }\end{array}$ & Included & $\begin{array}{c}\text { Prog } \\
\text { Transplant }\end{array}$ & $\begin{array}{l}\text { National } \\
\text { Library of } \\
\text { Medicine }\end{array}$ \\
\hline 22 & 20879013 & 2010 & $\begin{array}{l}\text { Gender disparity in } \\
\text { liver transplant } \\
\text { waiting-list } \\
\text { mortality: the } \\
\text { importance of } \\
\text { kidney function }\end{array}$ & $\begin{array}{c}\text { Original } \\
\text { Article }\end{array}$ & $\begin{array}{l}\text { Organ Procurement and } \\
\text { Transplantation Network } \\
\text { data for patients with end- } \\
\text { stage liver disease (ESLD) } \\
\text { on the waiting list who } \\
\text { were registered between } \\
\text { February } 2002 \text { and August } \\
2009.42,322 \text { patients and } \\
610,762 \text { person-months of } \\
\text { waiting-list experience were } \\
\text { included in the analysis. }\end{array}$ & Included & $\begin{array}{l}\text { Liver } \\
\text { transplantat } \\
\text { ion }\end{array}$ & $\begin{array}{l}\text { Wiley } \\
\text { Library }\end{array}$ \\
\hline
\end{tabular}




\begin{tabular}{|c|c|c|c|c|c|c|c|c|}
\hline 24 & 21668497 & 2011 & $\begin{array}{l}\text { Organ donation } \\
\text { from children: time } \\
\text { for legal, ethical } \\
\text { and cultural change }\end{array}$ & $\begin{array}{c}\text { Review } \\
\text { Article }\end{array}$ & $\begin{array}{c}\text { No age restriction as part of } \\
\text { the inclusion/exclusion } \\
\text { criteria. }\end{array}$ & Excluded & $\begin{array}{c}\text { Acta } \\
\text { Paediatr }\end{array}$ & $\begin{array}{l}\text { Wiley } \\
\text { Library }\end{array}$ \\
\hline 25 & 21426918 & 2011 & $\begin{array}{l}\text { Gender disparity } \\
\text { and MELD in liver } \\
\text { transplantation } \\
\end{array}$ & $\begin{array}{l}\text { Letter to } \\
\text { the Editor }\end{array}$ & $\begin{array}{l}\text { No inclusion/exclusion } \\
\text { criteria as part of the } \\
\text { research. }\end{array}$ & Excluded & $\begin{array}{c}\text { Journal of } \\
\text { Hepatol }\end{array}$ & $\begin{array}{l}\text { National } \\
\text { Library of } \\
\text { Medicine }\end{array}$ \\
\hline 27 & 23722379 & 2011 & $\begin{array}{l}\text { Organ donation } \\
\text { after circulatory } \\
\text { death: vital } \\
\text { partnerships }\end{array}$ & $\begin{array}{l}\text { Case } \\
\text { Report }\end{array}$ & $\begin{array}{l}\text { No inclusion/exclusion } \\
\text { criteria as part of the case } \\
\text { report. }\end{array}$ & Excluded & $\begin{array}{l}\text { The } \\
\text { American } \\
\text { Journal of } \\
\text { Nursing }\end{array}$ & $\begin{array}{l}\text { Wolters } \\
\text { Kluwer }\end{array}$ \\
\hline 28 & 23008117 & 2012 & $\begin{array}{l}\text { Impact of } \\
\text { estimated liver } \\
\text { volume and liver } \\
\text { weight on gender } \\
\text { disparity in liver } \\
\text { transplantation }\end{array}$ & $\begin{array}{l}\text { Original } \\
\text { Article }\end{array}$ & $\begin{array}{c}\text { The final cohort had } 28,866 \\
\text { patients who were analysed } \\
\text { depending on the Organ } \\
\text { Procurement and } \\
\text { Transplantation Network } \\
\text { Data as of August } 2009 .\end{array}$ & Included & $\begin{array}{l}\text { Liver } \\
\text { Transplanta } \\
\text { tion }\end{array}$ & $\begin{array}{l}\text { National } \\
\text { Library of } \\
\text { Medicine }\end{array}$ \\
\hline 32 & 25457096 & 2014 & $\begin{array}{l}\text { Organ donation by } \\
\text { suicides: sex and } \\
\text { ethnicity }\end{array}$ & $\begin{array}{l}\text { Original } \\
\text { Article }\end{array}$ & $\begin{array}{l}\text { An analysis of } 2,034 \text { actual } \\
\text { organ donations by suicides } \\
\text { for the years } 2008-2010\end{array}$ & Included & $\begin{array}{l}\text { Psychologic } \\
\text { al reports }\end{array}$ & $\begin{array}{l}\text { National } \\
\text { Library of } \\
\text { Medicine } \\
\end{array}$ \\
\hline 33 & 24996438 & 2014 & $\begin{array}{l}\text { Impact of gender } \\
\text { and professional } \\
\text { education on } \\
\text { attitudes towards } \\
\text { financial incentives } \\
\text { for organ donation: } \\
\text { results of a survey } \\
\text { among } 755 \\
\text { students of } \\
\text { medicine and } \\
\text { economics in } \\
\text { Germany }\end{array}$ & $\begin{array}{l}\text { Original } \\
\text { Article }\end{array}$ & $\begin{array}{l}\text { Between October } 2008 \text { and } \\
\text { February } 2009 \text {, a } \\
\text { quantitative survey was } \\
\text { conducted among German } \\
\text { students of medicine and } \\
\text { economics }\end{array}$ & Included & $\begin{array}{l}\text { BMC } \\
\text { Medical } \\
\text { Ethics }\end{array}$ & $\begin{array}{l}\text { National } \\
\text { Library of } \\
\text { Medicine }\end{array}$ \\
\hline 34 & 26413291 & 2015 & $\begin{array}{l}\text { A peer outreach } \\
\text { initiative to } \\
\text { increase the } \\
\text { registration of } \\
\text { minorities as organ } \\
\end{array}$ & $\begin{array}{l}\text { Original } \\
\text { Article }\end{array}$ & $\begin{array}{c}\text { A peer outreach } \\
\text { programme piloted in } \\
\text { Harrow (Middlesex) in } \\
2009 \text { and later rolled out to } \\
\text { Hounslow, Lewisham and }\end{array}$ & Included & $\begin{array}{l}\text { Clinical } \\
\text { Kidney } \\
\text { Journal }\end{array}$ & $\begin{array}{l}\text { Oxford } \\
\text { Academic }\end{array}$ \\
\hline
\end{tabular}




\begin{tabular}{|c|c|c|c|c|c|c|c|c|}
\hline & & & donors & & $\begin{array}{c}\text { Lambeth in } 2010 \text { (all in } \\
\text { London, UK) was taken as } \\
\text { the superset. }\end{array}$ & & & \\
\hline 35 & 26465666 & 2015 & $\begin{array}{l}\text { Gender in the } \\
\text { allocation of organs } \\
\text { in kidney } \\
\text { transplants: meta- } \\
\text { analysis }\end{array}$ & $\begin{array}{l}\text { Review } \\
\text { article }\end{array}$ & $\begin{array}{c}\text { Twenty-nine studies } \\
\text { involving } 765,753 \text { patients } \\
\text { were included. }\end{array}$ & Included & $\begin{array}{l}\text { Revista de } \\
\text { saude } \\
\text { publica }\end{array}$ & $\begin{array}{l}\text { National } \\
\text { Library of } \\
\text { Medicine }\end{array}$ \\
\hline 38 & 27471591 & 2016 & $\begin{array}{c}\text { Organ } \\
\text { transplantation and } \\
\text { gender differences: } \\
\text { a paradigmatic } \\
\text { example of } \\
\text { intertwining } \\
\text { between biological } \\
\text { and sociocultural } \\
\text { determinants }\end{array}$ & $\begin{array}{l}\text { Comment } \\
\text { ary }\end{array}$ & $\begin{array}{l}\text { No inclusion/exclusion } \\
\text { criteria as part of the } \\
\text { commentary. }\end{array}$ & Included & $\begin{array}{l}\text { Biology of } \\
\text { Sex } \\
\text { Differences }\end{array}$ & $\begin{array}{l}\text { National } \\
\text { Library of } \\
\text { Medicine }\end{array}$ \\
\hline 39 & 27015531 & 2016 & $\begin{array}{l}\text { Gender Disparity } \\
\text { in Living-Donor } \\
\text { Kidney Transplant } \\
\text { Among Minority } \\
\text { Ethnic Groups } \\
\end{array}$ & $\begin{array}{l}\text { Original } \\
\text { Article }\end{array}$ & $\begin{array}{l}\text { analysed } 713 \text { living-donor } \\
\text { kidney transplant } \\
\text { procedures that were } \\
\text { performed from } 1987 \text { to } \\
2014 . \\
\end{array}$ & Included & $\begin{array}{l}\text { Experiment } \\
\text { al and } \\
\text { Clinical } \\
\text { Transplanta } \\
\text { tion } \\
\end{array}$ & $\begin{array}{l}\text { National } \\
\text { Library of } \\
\text { Medicine }\end{array}$ \\
\hline 41 & 29284769 & 2017 & $\begin{array}{l}\text { Few Gender } \\
\text { Differences in } \\
\text { Attitudes and } \\
\text { Experiences after } \\
\text { Live Kidney } \\
\text { Donation, with } \\
\text { Minor Changes } \\
\text { Over Time }\end{array}$ & $\begin{array}{l}\text { Original } \\
\text { Article }\end{array}$ & $\begin{array}{c}455 \text { live kidney donors } \\
\text { between } 1974 \text { and } 2008 \\
\text { were considered for this } \\
\text { study. }\end{array}$ & Included & $\begin{array}{l}\text { Annals of } \\
\text { Transplnt }\end{array}$ & $\begin{array}{l}\text { National } \\
\text { Library of } \\
\text { Medicine }\end{array}$ \\
\hline 42 & 27352118 & 2017 & $\begin{array}{c}\text { Assessing } \\
\text { Transplant } \\
\text { Attitudes: } \\
\text { Understanding } \\
\text { Minority Men's } \\
\text { Perspectives on the } \\
\text { Multifarious } \\
\text { Barriers to Organ } \\
\text { Donation }\end{array}$ & $\begin{array}{l}\text { Original } \\
\text { Article }\end{array}$ & $\begin{array}{l}\text { A } 57 \text { question Health and } \\
\text { Wellness survey was } \\
\text { designed to assess } \\
\text { participants' demographic } \\
\text { information, medical } \\
\text { history, professional } \\
\text { background, and opinions } \\
\text { about organ } \\
\text { transplantation. } \\
\text { Participants were also } \\
\text { asked to complete Quality } \\
\text { Metric's Short Form-8 (SF- } \\
\text { 8) survey to assess physical } \\
\text { health, mental health, and } \\
\text { quality-of-life. Three }\end{array}$ & Included & $\begin{array}{l}\text { Journal of } \\
\text { Racial \& } \\
\text { Ethnic } \\
\text { Health } \\
\text { Disparities. }\end{array}$ & $\begin{array}{l}\text { National } \\
\text { Library of } \\
\text { Medicine }\end{array}$ \\
\hline
\end{tabular}




\begin{tabular}{|c|c|c|c|c|c|c|c|c|}
\hline & & & & & $\begin{array}{l}\text { hundred twenty-six surveys } \\
\text { were administered to } \\
\text { minority men. }\end{array}$ & & & \\
\hline 43 & 28540899 & 2017 & $\begin{array}{l}\text { Gender differences } \\
\text { in perceptions and } \\
\text { attitudes of general } \\
\text { population towards } \\
\text { organ donation: An } \\
\text { Indian perspective }\end{array}$ & $\begin{array}{l}\text { Original } \\
\text { Article }\end{array}$ & $\begin{array}{l}\text { A cross-sectional } \\
\text { descriptive study was } \\
\text { conducted among } \\
\text { randomly selected patient } \\
\text { relatives }(\mathrm{n}=193) \text { at the } \\
\text { outpatient department of a } \\
\text { tertiary care center. }\end{array}$ & Included & $\begin{array}{l}\text { Saudi } \\
\text { journal of } \\
\text { kidney } \\
\text { diseases } \\
\text { and } \\
\text { transplantat } \\
\quad \text { ion }\end{array}$ & $\begin{array}{l}\text { National } \\
\text { Library of } \\
\text { Medicine }\end{array}$ \\
\hline 46 & 30587146 & 2018 & $\begin{array}{l}\text { Gender disparity in } \\
\text { health-related } \\
\text { quality of life and } \\
\text { fatigue after living } \\
\text { renal donation }\end{array}$ & $\begin{array}{l}\text { Original } \\
\text { Article }\end{array}$ & $\begin{array}{c}\text { Two hundred and eleven } \\
\text { (211) living renal donors } \\
\text { were evaluated (female } \\
62.2 \%)\end{array}$ & Included & $\begin{array}{c}\text { BMC } \\
\text { Nephrology }\end{array}$ & $\begin{array}{l}\text { National } \\
\text { Library of } \\
\text { Medicine }\end{array}$ \\
\hline 49 & 30213924 & 2018 & $\begin{array}{l}\text { Factors in Organ } \\
\text { Donation } \\
\text { Coordinators: A } \\
\text { Cross-Sectional } \\
\text { Study in China } \\
\end{array}$ & $\begin{array}{l}\text { Original } \\
\text { Article }\end{array}$ & $\begin{array}{l}\text { From March to May 2017, } \\
\text { we administered } \\
\text { questionnaire surveys to } \\
320 \text { organ donation } \\
\text { coordinators from } 32 \text { cities. }\end{array}$ & Excluded & $\begin{array}{l}\text { Annals of } \\
\text { transplantat } \\
\text { ion }\end{array}$ & $\begin{array}{l}\text { National } \\
\text { Library of } \\
\text { Medicine }\end{array}$ \\
\hline 50 & 31929292 & 2019 & $\begin{array}{l}\text { Women donate, } \\
\text { men receive gender } \\
\text { disparity among } \\
\text { renal donors }\end{array}$ & $\begin{array}{l}\text { Original } \\
\text { Article }\end{array}$ & $\begin{array}{l}\text { A single centre, } \\
\text { retrospective cohort study } \\
\text { on } 600 \text { kidney donors } \\
\text { between } 2013 \text { and } \\
\text { November } 2018\end{array}$ & Included & $\begin{array}{l}\text { Saudi } \\
\text { journal of } \\
\text { kidney } \\
\text { diseases } \\
\text { and } \\
\text { transplantat } \\
\quad \text { ion }\end{array}$ & $\begin{array}{l}\text { Wolters } \\
\text { Kluwer }\end{array}$ \\
\hline 51 & 30105865 & 2019 & $\begin{array}{l}\text { Gender dynamics } \\
\text { in the donation } \\
\text { field: human tissue } \\
\text { donation for } \\
\text { research, therapy } \\
\text { and feeding }\end{array}$ & $\begin{array}{c}\text { Review } \\
\text { Article }\end{array}$ & $\begin{array}{l}\text { No inclusion/exclusion } \\
\text { criteria as part of the } \\
\text { review. }\end{array}$ & Included & $\begin{array}{l}\text { Sociology } \\
\text { of Health } \\
\text { and Illness }\end{array}$ & $\begin{array}{l}\text { National } \\
\text { Library of } \\
\text { Medicine }\end{array}$ \\
\hline 52 & 30747857 & 2019 & $\begin{array}{c}\text { Equally } \\
\text { Interchangeable? } \\
\text { How Sex and } \\
\text { Gender Affect } \\
\text { Transplantation }\end{array}$ & $\begin{array}{c}\text { Review } \\
\text { Article }\end{array}$ & $\begin{array}{l}\text { No inclusion/exclusion } \\
\text { criteria as part of the } \\
\text { review. }\end{array}$ & Included & $\begin{array}{c}\text { Transplanta } \\
\text { tion }\end{array}$ & $\begin{array}{l}\text { Wolters } \\
\text { Kluwer }\end{array}$ \\
\hline 53 & 30384800 & 2019 & A care ethics & Original & No inclusion/exclusion & Included & Nursing & Sage \\
\hline
\end{tabular}




\begin{tabular}{|c|c|c|c|c|c|c|c|c|}
\hline & & & $\begin{array}{l}\text { approach to the } \\
\text { Gender Kidney } \\
\text { Donation Gap }\end{array}$ & Article & criteria as part of the article. & & Ethics & \\
\hline 54 & 31518477 & 2019 & $\begin{array}{l}\text { In sickness and in } \\
\text { health: Living HIV } \\
\text { positive kidney } \\
\text { donation from a } \\
\text { wife to her } \\
\text { husband, with } 7 \\
\text { years' post- } \\
\text { transplant follow- } \\
\text { up }\end{array}$ & $\begin{array}{l}\text { Case } \\
\text { Report }\end{array}$ & $\begin{array}{l}\text { No inclusion/exclusion } \\
\text { criteria as part of the case } \\
\text { report. }\end{array}$ & Excluded & $\begin{array}{l}\text { Transplant } \\
\text { Infectious } \\
\text { Disease }\end{array}$ & $\begin{array}{l}\text { Wiley } \\
\text { Library }\end{array}$ \\
\hline 55 & 31461744 & 2019 & $\begin{array}{c}\text { Sex and Gender } \\
\text { Considerations in } \\
\text { Transplant } \\
\text { Research: A } \\
\text { Scoping Review }\end{array}$ & $\begin{array}{l}\text { Review } \\
\text { Article }\end{array}$ & $\begin{array}{l}\text { No inclusion/exclusion } \\
\text { criteria as part of the } \\
\text { review. }\end{array}$ & Included & $\begin{array}{c}\text { Transplanta } \\
\text { tion }\end{array}$ & $\begin{array}{l}\text { Wolters } \\
\text { Kluwer }\end{array}$ \\
\hline 56 & 30777567 & 2019 & $\begin{array}{l}\text { Gender Disparity } \\
\text { and the } \\
\text { Relationship } \\
\text { Between Living } \\
\text { Donors and } \\
\text { Recipients in } \\
\text { Kidney Transplants } \\
\text { in an Organ } \\
\text { Transplant Center } \\
\text { in Turkey }\end{array}$ & $\begin{array}{c}\text { Original } \\
\text { Article }\end{array}$ & $\begin{array}{c}\text { Analysed the hospital } \\
\text { records of living kidney } \\
\text { donors (1611 cases) and all } \\
1991 \text { kidney recipients who } \\
\text { underwent living-donor and } \\
\text { deceased-donor kidney } \\
\text { transplant procedures in a } \\
\text { university hospital between } \\
1985 \text { and } 2017 .\end{array}$ & Included & $\begin{array}{l}\text { Experiment } \\
\text { al and } \\
\text { Clinical } \\
\text { Transplanta } \\
\text { tion }\end{array}$ & $\begin{array}{l}\text { National } \\
\text { Library of } \\
\text { Medicine }\end{array}$ \\
\hline 57 & 30879523 & 2019 & $\begin{array}{l}\text { Attitude Toward } \\
\text { Organ Donation in } \\
\text { the Population of } \\
\text { Cienfuegos, Cuba }\end{array}$ & $\begin{array}{c}\text { Original } \\
\text { Article }\end{array}$ & $\begin{array}{l}\text { Population over } 15 \text { years } \\
\text { old as stratified by age and } \\
\text { sex. }\end{array}$ & Excluded & $\begin{array}{l}\text { Transplanta } \\
\text { tion } \\
\text { Proceeding }\end{array}$ & $\begin{array}{l}\text { National } \\
\text { Library of } \\
\text { Medicine }\end{array}$ \\
\hline 58 & 31533778 & 2019 & $\begin{array}{l}\text { An ethical } \\
\text { comparison of } \\
\text { living kidney } \\
\text { donation and } \\
\text { surrogacy: } \\
\text { understanding the } \\
\text { relational } \\
\text { dimension }\end{array}$ & $\begin{array}{c}\text { Review } \\
\text { Article }\end{array}$ & $\begin{array}{l}\text { No inclusion/exclusion } \\
\text { criteria as part of the } \\
\text { review. }\end{array}$ & Excluded & $\begin{array}{l}\text { Philosophy, } \\
\text { ethics \& } \\
\text { humanity } \\
\text { in medicine }\end{array}$ & $\begin{array}{l}\text { National } \\
\text { Library of } \\
\text { Medicine }\end{array}$ \\
\hline 59 & 31906885 & 2020 & $\begin{array}{c}\text { Impact of the } \\
\text { donor-recipient } \\
\text { gender matching } \\
\text { on the graft } \\
\text { survival from live } \\
\text { donors } \\
\end{array}$ & $\begin{array}{c}\text { Original } \\
\text { Article }\end{array}$ & $\begin{array}{l}1113 \text { kidney transplant } \\
\text { recipients were studied in } \\
\text { this retrospective cohort } \\
\text { study. }\end{array}$ & Included & $\begin{array}{c}\text { BMC } \\
\text { Nephrology }\end{array}$ & BMC \\
\hline 60 & 11802081 & 2020 & $\begin{array}{c}\text { Understanding } \\
\text { disparities in donor } \\
\text { behaviour: race and } \\
\text { gender differences } \\
\text { in willingness to } \\
\text { donate blood and } \\
\text { cadaveric organs }\end{array}$ & $\begin{array}{c}\text { Original } \\
\text { Article }\end{array}$ & $\begin{array}{l}\text { Cross-sectional telephone } \\
\text { survey of persons aged } 18 \\
\text { to } 75 \text { living in the } \\
\text { Baltimore, Maryland } \\
\text { metropolitan area contacted } \\
\text { via random digit dialling. }\end{array}$ & Included & $\begin{array}{l}\text { Medical } \\
\text { Care }\end{array}$ & $\begin{array}{l}\text { Wolters } \\
\text { Kluwer }\end{array}$ \\
\hline
\end{tabular}

From the above taken 60 studies via the database, a total of 47 studies met all the inclusion criteria and were selected following the PRISMA (Preferred Reporting Items for Systematic Reviews and Meta-Analyses) guideline [6]. A comprehensive outline for the method of selection is as given in Figure 1. 


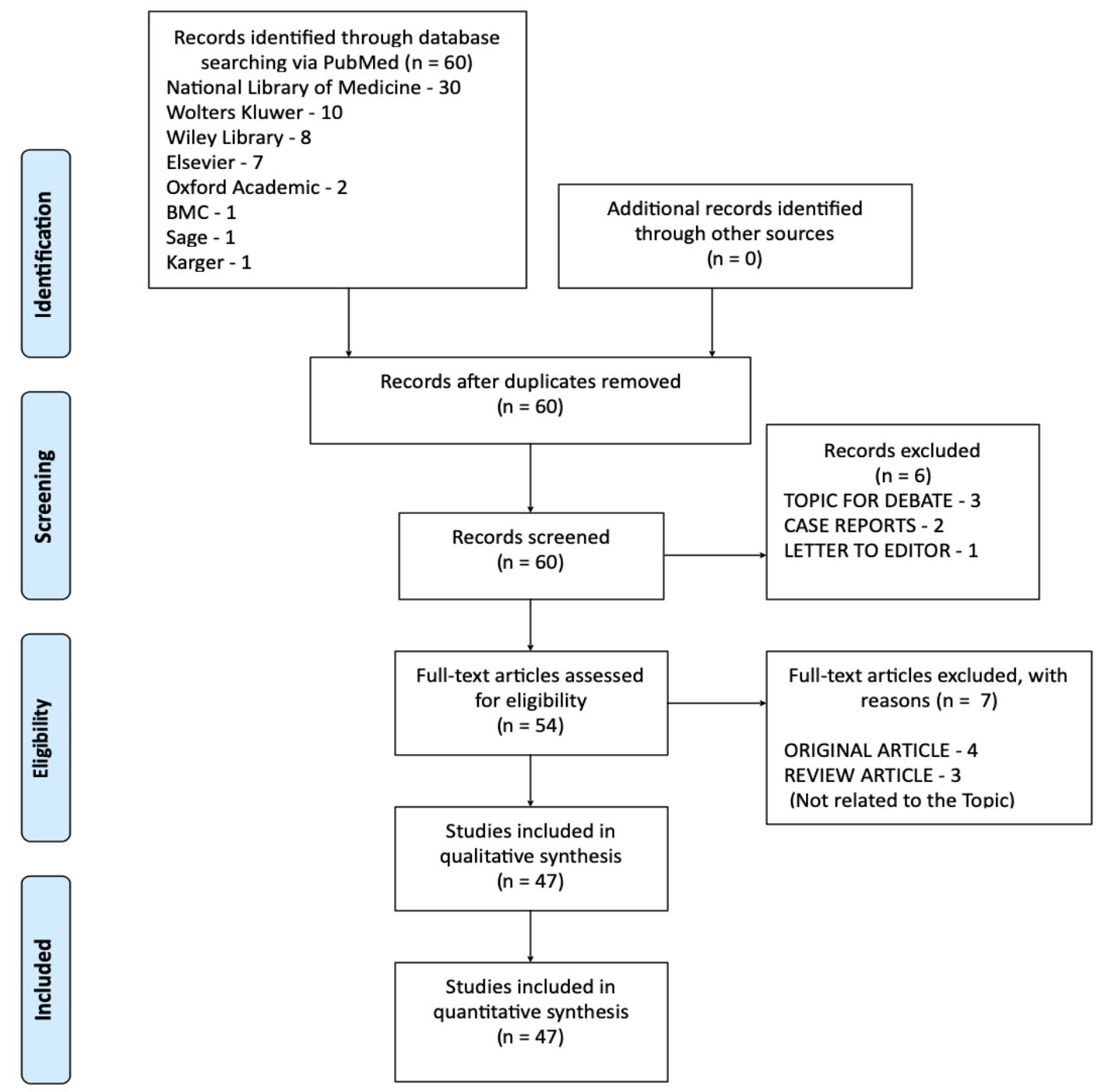

Figure 1: PRISMA Flow Diagram for Selection of Review Material

\section{Results}

Through our review, we found that there exists a disparity between men and women in the context of organ donation, which is further substantiated by the fact that not only do women tend to donate more, but also that men are more predominantly the recipients [7-8]. This disparity transcends all relations, in that spousal as well as parental donations are from women to men [9-12]. As for female recipient numbers, they could be $38 \%$ vs $62 \%$ for males [13], or as skewed as $11 \%$ vs $89 \%$ for males [14]. Nevertheless, females predominantly donating and males predominantly receiving is a universal trend. This, despite males and females having a level playing field in terms of getting through the required steps to be approved as a donor. However, among approved potential donors, females have shown more likelihood to donate [15] and are also more likely than men to agree to donate their organs, live or otherwise [16].

\section{Organs and organ systems}

organ donation patterns have almost universally highlighted a propensity for females to play the role of live donors [17]. This could be as simple as donation of blood [18] or as complex as donation of liver and kidney [19-20]. According to a study done in the United States, women had a 25\% lesser chance of receiving a liver transplant, compared to male recipients, in any given month, coming with increasing the likelihood of women dying while waitlisted [21-22]. The same applies for spousal kidney donations; they are more likely to be from wife to husband [19-20]. 


\section{Role of gender in graft rejection}

Female-donated livers come with a higher risk of graft rejection, however it is not known to what degree such findings influence transplantation of other organs like the heart, lung or kidneys [23]. Overall, the findings tend to confirm that genders of the donor and recipient influences the recipient survival, thus exacerbating the already prevalent gender bias in organ transplantation and donation [24]. In one such example, boys in a study of South-Asian study received only the kidneys donated by men [2] despite there being no concrete evidence to support such practice [23], as if pre-emptive measure to reduce the chances of graft rejection on account of the difference in gender between donor and recipient.

Age

There is a palatable disparity in most transplantations of kidney, liver, lung, heart and, in some cases, even hematopoietic transplantations be it in adult or paediatric age-group cases [25]. In a study, we also discovered that in the cases where men agree to be donors, they do so more readily for their children than for their wives [2]. Donations to children have not been as skewed towards males as donations among adults, with women having as much as $11 \%$ lesser chance of receiving a transplant, the ratio becoming more skewed in case of specific races or organs $[22,26]$. Nevertheless, there is a propensity for male and younger recipients to be favoured, while in case of donors, females and middle-aged or older individuals are preferred [27-28]. This suggests that the engulfing by stereotypes and psycho-socio-economic factors, of organ donation and transplantation, occurs nearer to the human "middle age" [26].

\section{Social Relations}

It is no secret now that societal factors have been mirrored in the prevalent disparity in organ donation between the two binary genders. With a majority of donations being between those related by blood, it is ever more important to examine exactly how much the prevailing systems burden women [8]. Women themselves are somewhat reluctant to be recipients of an organ [23] but are astonishingly high in number among those who hold a donor card [5]. In a study of South Asian spousal transplants, the majority of the donations were done from wife to the husband, which has been the trend in all the studies reviewed. In the same study, it was found that children were donated to equitably irrespective of their gender. However, boys received only those kidneys that were donated by men [2].

\section{Medical factors}

Multiple studies under our review mention the probable iatrogenic factors leading to this disparity in organ donation. Several studies mentioned how, in case of liver transplants, the Model for Endstage Liver Disease (MELD) Score places women at a significant disadvantage as the scores are based on serum creatinine levels, without taking into account that women have physiologically lower serum creatinine levels than their male counterparts [29-30]. In another comparison of men and women on the waitlist for a liver transplant, women were generally less favoured on the grounds of their short stature leading to increased transplantation failure [3]. Besides, the probable and quite accounted for gender bias among medical professionals and their work environment might also be a significant contributor [31].

\section{Race, religion, and culture}

It is documented that while women tend to donate more than men overall, White individuals donate more than other races, especially more than Black individuals [32-33] while a study reported that in the United States, Asian-Americans make up the least number of registered donors [34]. In a study of race-gender groups, Black women donated blood the least, while Black men were most reluctant for themselves to be donated as cadavers [35]. This can be attributed to a wellknown mistrust of minorities in general and Black communities in particular in the medical system and their perception of the profession, and there is a very strong correlation between race and the unwillingness to donate organs [36]. Concerns also remain among individuals about the acceptance of organ donation in their religion [16], and the desire to be buried intact [37]. As for 
certain Asian races, a study conducted in China reported that $34.4 \%$ expressed willingness to donate organs, but that the overwhelming majority of respondents showed concerns about appropriate use and legislation, ranging between $61.4 \%$ and $88.9 \%$ depending on the factors considered, while overwhelming favouring consideration of family opinions [38]. Meanwhile, in India, some religious and linguistic communities are more altruistic than others, as seen in Mumbai, where $85 \%$ of eye donations and $95 \%$ of skin donations in 2013 and $70 \%$ of the cadaver donations recorded in 2016 were from the Jain and Gujarati communities, which have also been known to be leaders in the donation of blood, per Mumbai's Zonal Transplant Coordination Committee [39]. However, misconceptions such as one kidney being insufficient for survival and fear of the operation exist in various Asian communities, preventing the widespread acceptance of such practices [40].

\section{Economic perspective}

There is a growing consensus that the shroud of altruism is one of the reasons behind the demandsupply chasm in organ donation, especially of the deceased type, and a lot of organs that could be harvested are missed out on, irrespective of reason [41]. Besides, the idea of altruism and summary bans on commercial transplants have led to factors conducive for monetisation of organ transplantation [42] illegally and exploitatively. Aside from this, certain communities, majorly the Black community, do agree more to subsidies, if not incentives, for deceased organ donors' funeral expenses to be covered, for example [36], especially among men [5]. Even in Chinese communities, $64.5 \%$ respondents agreed on compensation for donors, while $74.4 \%$ agreed that "Donated organs have not been fairly and appropriately used; the wealthy and celebrities have been favoured [38].

\section{Discussion}

There have been professed to be a multitude of factors for the disparity, as described earlier, but the fact remains: women tend to play the role of donors more than they do of recipients, and the problem can only be surmounted through a collective effort by society to carefully analyse and make aware of said factors and commonly tackle them. Women, intrinsically, have been known to hold greater emotional quotient [43] and have an inherently more profound sense of responsibility [7] as their biological role as child bearers and carers. This same sense of responsibility, even in the modern world, leads to their greater self-sacrifice-which might explain their reluctance to undergo transplant surgery[23] and their care not just directed towards the health and longevity of their children, but also of their spouses an idea that has even found place and reasoning in certain religions and prayer. Women have also shown greater application of scientific thought, in that a study found that women hold lesser superstitions and other disgust about organ donation while also not being too keen on the idea of bodies being intact during last rites.[44] This may lead to an inherent bias in women taking on the role of donors, especially of their own volition. Thus, it could be hypothesised that women are inherently more willing to donate organs than men.

However, factors brought in by societal thought and sociocultural relations contribute to an exacerbation of this bias: In another study, it was observed that the transplants most suitable for survival were either male donor to male recipient or male donor to female recipient. [45] This in the light of two observations, one being from the aforementioned study of South-Asians and the other being that women still bear the burden of donating, suggest that the vitality and life and health of males is preferred by society, over females. This is compounded by the universally greater income of men compared to women doing the same work that may place the job of organ donation on women to ensure the breadwinner's health is not compromised. Adding to this, a gender bias among medical professionals [31], that normally extends in a few other fields [46-48], thereby increasing the already inherent gap.

Even in parameters made to assess the need for a transplant, the physiological reasons behind better scores for women remain unaccounted for [21,30,49], thus making them appear healthier and subsequently increasing their time on the waitlist, leading to women dying on the waitlist far more than men [3]. This, coupled with an inequitable access to medical care for women of all ages [25], including but not limited to organ transplantation [25], serves to further widen the gap. 
As for racial factors, there remains a deep-rooted historical mistrust in the medical profession [16], of non-white races in general, and Black communities in particular [50-53]. Multiple studies have highlighted the inequitable access of Black women to, for example, pain medications/epidural during delivery [54] as doctors, especially white doctors and medical trainees, tend to wrongly believe that black women have a higher tolerance to pain [55]. As for Black men, the history of their ancestors being used for medical trials without proper information, consent and ancillary care has caused their mistrust in the medical community [56], to a point where they believe "... if doctors know I am an organ donor, they won't try to save my life..." [36]. Nevertheless, here too, women tend to suffer more and as all these factors, along with a perceived inequity on who the organs will go to [36], transcends into organ transplantation and donation, so does the community. However, there was consensus on the willingness to donate a loved one's organs [36] and that no relative should override a deceased donor's wish to donate [57]. Meanwhile, in India, relatives have been known to override a deceased person's wish and express consent to donate, and superstitions about organ donation being sinful, belief in rebirth especially that intact bodies are required for rebirth and acceptance into heaven exist in India. This is known to be one of the major reasons why only $1 \%$ of Indians consent to organ donation after death, as compared to $70-80 \%$ of Western populations, as per reports from non-governmental organisations (NGOs). It has been widely suggested that religious leaders be roped into popular discourse to dispel a lot of the superstition, as religion has been known to play a key role in influencing followers towards or away from organ donation, be it live or cadaver donations [58].

As for economics, there is no doubt that organ transplant has blossomed into an often-illegal trade, thanks to the principle of altruism that is normally followed, and the lucrative money-making options in the field, leading to people resorting to illegal and unethical means to obtain organs. This may cause poorer girls and women to be forcefully made to donate organs, either by financial conditions or subjugation and suppression by men [1]. However, doubts linger as to whether direct incentivisation should be allowed or indirect benefits [5], for example the coverage of funeral expenses, should be provided. Consensus is split among gender and racial lines [5]. Nevertheless, it is agreed that relying solely on altruism is insufficient to bridge the demand-supply gap and target illegal organ trade [41].

It is therefore abundantly clear that participation will be needed from all levels of society, be it from the individual or from government. The initiative could come from anywhere and in any form, but it shall require a concerted effort from all levels with equal gusto. At the individual level, one could start by scoping for and identifying the various socio-cultural and psychological factors behind the prevailing gender gap in organ transplantation and donation and assimilating them, while also raising awareness among their peers, family and friends and gauging their attitudes and beliefs [59]. The awareness-building could be elevated to a broader level by the efforts of the community, while community organisations can mobilise efforts to assess economic factors as well, to encourage social support organisations especially for women and to encourage "healthseeking behaviours" in women [31] and to provide, per a study, "... Improving the general, physical, and mental health of minorities, coupled with an active educational outreach program.." in case of minorities, as it "...could result in a greater percentage of minorities registering and willing to be organ and tissue donors [60]." As for the medical profession, more needs to be done to repair an often tested relationship with the marginalised and underprivileged, be it in terms of race, financial background or otherwise. The beginning is to revisit the misconceptions plaguing an otherwise scientific profession and coming to terms with our checkered past, for that is the best way to begin to unlearn all falsehoods and through education, community engagement and empathy, can we regain lasting trust of all in the medical fraternity. More theoretically, tests or scores used to assess the need for organs, like the MELD Score, and in general, should account for the biological and physiological differences between the genders. Finally, at the level of government, we need a broad relook at the various legislations governing organ donation in each country and, where necessary, amend existing laws to influence the nations' citizens towards a more informed polity and encourage scientific temper, while also ensuring accountability, transparency and due recourse for any illegalities or immoral transgressions. 
It must be acknowledged that no one person, party or platform can effectively bring about lasting reforms unless aided and in cohesion with every element of society, free of bias and personal stake.

\section{Conclusion}

Gender disparity in organ donation exists throughout the world. Wives to husbands, mothers to sons and sisters to brothers women have been obeying the gender roles enforced on them for ages. It indeed is a challenge to go beyond the gender gap to make the domain of organ transplant impartial, equitable and just, but it remains the need of the hour if we are to ensure equitable healthcare and healthy living for all, regardless of their gender. Inequitable and immoral practices have personal and ethical implications and need to be eliminated to advance our goals of equality and justice as a basic tenet of respect for every human life and to advance further as a civilised society.

\section{REFERENCES}

1. Steinman JL. Gender disparity in organ donation. Gend Med. 2006;3(4):246-52.

2. Peracha J, Hayer MK, Sharif A. Gender Disparity in Living-Donor Kidney Transplant Among Minority Ethnic Groups. Exp Clin Transplant 2016;14(2):139-45.

3. Nephew LD, Goldberg DS, Lewis JD, Abt P, Bryan M, Forde KA. Exception Points and Body Size Contribute to Gender Disparity in Liver Transplantation. Clin Gastroenterol Hepatol 2017;15(8):1286-1293.e2.

4. Thornton JD, Wong KA, Cardenas V, Curtis JR, Spigner C, Allen MD. Ethnic and gender differences in willingness among high school students to donate organs. J Adolesc Health 2006;39(2):266-74.

5. Inthorn J, Wöhlke S, Schmidt F, Schicktanz S. Impact of gender and professional education on attitudes towards financial incentives for organ donation: results of a survey among 755 students of medicine and economics in Germany. BMC Med Ethics 2014;15:56.

6. Moher D, Liberati A, Tetzlaff J, Altman DG, Altman D, Antes G, et al. Preferred reporting items for systematic reviews and meta-analyses: the PRISMA statement. Ann Intern Med 2009;151(4):264-9.

7. Puoti F, Ricci A, Nanni-Costa A, Ricciardi W, Malorni W, Ortona E. Organ transplantation and gender differences: a paradigmatic example of intertwining between biological and sociocultural determinants. Biol Sex Differ 2016;7:35.

8. Hermann HC, Klapp BF, Danzer G, Papachristou C. Gender-specific differences associated with living donor liver transplantation: a review study. Liver Transpl 2010;16(3):375-86.

9. Zimmerman D, Donnelly S, Miller J, Stewart D, Albert SE. Gender disparity in living renal transplant donation. Am J Kidney Dis 2000;36(3):534-40.

10. Kayler LK, Meier-Kriesche HU, Punch JD, Campbell DA, Leichtman AB, Magee JC, et al. Gender imbalance in living donor renal transplantation. Transplantation 2002;73(2):248-52.

11. Sehgal AR. The net transfer of transplant organs across race, sex, age, and income. Am J Med 2004;117(9):670-5.

12. Øien CM, Reisaeter AV, Leivestad T, Pfeffer P, Fauchald P, Os I. Gender imbalance among donors in living kidney transplantation: the Norwegian experience. Nephrol Dial Transplant 2005;20(4):783-9.

13. Mohsin N, Budruddin M, Khalil M, Pakkyarra A, Jha A, Mohammed E, et al. Donor gender balance in a living-related kidney transplantation program in Oman. Transplant Proc 2007;39(4):803-6.

14. Bal MM, Saikia B. Gender bias in renal transplantation are women alone donating kidneys in India. Transplant Proc 2007;39(10):2961-3.

15. Tuohy KA, Johnson S, Khwaja K, Pavlakis M. Gender disparities in the live kidney donor evaluation process. Transplantation 2006;82(11):1402-7.

16. Breitkopf CR. Attitudes, beliefs and behaviors surrounding organ donation among Hispanic women. Curr Opin Organ Transplant 2009;14(2):191-5.

17. Csete M. Gender issues in transplantation. Anesth Analg 2008;107(1):232-8.

18. Kent J, Fannin M, Dowling S. Gender dynamics in the donation field: human tissue donation for research, therapy and feeding. Sociol Health Illness 2019;41(3):567-84.

19. Godara S, Jeswani J. Women donate, men receive gender disparity among renal donors. Saudi J 
Kidney Dis Transpl 2019;30(6):1439-41.

20. Hodson N. A care ethics approach to the Gender Kidney Donation Gap. Nurs Ethics 2019;26(78):2185-94.

21. Nephew LD, Goldberg DS, Lewis JD, Abt P, Bryan M, Forde KA. Exception Points and Body Size Contribute to Gender Disparity in Liver Transplantation. Clin Gastroenterol Hepatol 2017;15(8):1286-93.

22. Mindikoglu AL, Emre SH, Magder LS. Impact of estimated liver volume and liver weight on gender disparity in liver transplantation. Liver Transpl 2013;19(1):89-95.

23. Ge F, Huang T, Yuan S, Zhou Y, Gong W. Gender issues in solid organ donation and transplantation. Ann Transplant 2013;18:508-14.

24. Sanfey H. Gender-specific issues in liver and kidney failure and transplantation: a review. J Womens Health (Larchmt) 2005;14(7):617-26.

25. Melk A, Babitsch B, Borchert-Mörlins B, Claas F, Dipchand AI, Eifert S, et al. Equally Interchangeable? How Sex and Gender Affect Transplantation. Transplantation 2019;103(6):1094110.

26. Segev DL, Kucirka LM, Oberai PC, Parekh RS, Boulware LE, Powe NR, et al. Age and comorbidities are effect modifiers of gender disparities in renal transplantation. J Am Soc Nephrol 2009;20(3):621-8.

27. Liu G, Li X, Liu T, Zhao X, Zhang S, Wang J, et al. Gender disparity of living donor renal transplantation in East China. Clin Transplant 2013;27(1):98-103.

28. Mihçıokur S, Ayvazoğlu Soy EH, Türkçelik E, Akın A, Haberal M. Gender Disparity and the Relationship Between Living Donors and Recipients in Kidney Transplants in an Organ Transplant Center in Turkey. Exp Clin Transplant 2019;17(Suppl 1):246-9.

29. Hermann HC, Klapp BF, Danzer G, Papachristou C. Gender-specific differences associated with living donor liver transplantation: a review study. Liver Transpl 2010;16(3):375-86.

30. Cholongitas E, Thomas M, Senzolo M, Burroughs AK. Gender disparity and MELD in liver transplantation. J Hepatol 2011;55(2):500-1.

31. Jindal RM, Ryan JJ, Sajjad I, Murthy MH, Baines LS. Kidney transplantation and gender disparity. Am J Nephrol 2005;25(5):474-83.

32. Thornton JD, Wong KA, Cardenas V, Curtis JR, Spigner C, Allen MD. Ethnic and gender differences in willingness among high school students to donate organs. J Adolesc Health 2006;39(2):266-74.

33. Lester D, Hathaway D. Organ donation by suicides: sex and ethnicity. Psychol Rep 2014;115(3):948-50.

34. Li MT, Hillyer GC, Husain SA, Mohan S. Cultural barriers to organ donation among Chinese and Korean individuals in the United States: a systematic review. Transpl Int 2019;32(10):1001-18.

35. Boulware LE, Ratner LE, Cooper LA, Sosa JA, LaVeist TA, Powe NR. Understanding disparities in donor behavior: race and gender differences in willingness to donate blood and cadaveric organs. Med Care 2002;40(2):85-95.

36. Siminoff LA, Burant CJ, Ibrahim SA. Racial disparities in preferences and perceptions regarding organ donation. J Gen Intern Med 2006;21(9):995-1000.

37. Edwards TM, Essman C, Thornton JD. Assessing racial and ethnic differences in medical student knowledge, attitudes and behaviors regarding organ donation. J Natl Med Assoc 2007;99(2):131-7.

38. Aijing L, Wenzhao X, Wei W, Qiquan W, Xuantong D. Public Opinion on Organ Donation After Death and Its Influence on Attitudes Toward Organ Donation. Ann Transplant 2016;21:516-24.

39. http://ztccmumbai.org/

40. Mohamed E, Guella A. Public awareness survey about organ donation and transplantation. Transplant Proc 2013;45(10):3469-71.

41. Omar F, Tinghög G, Welin S. Incentivizing deceased organ donation: a Swedish priority-setting perspective. Scand J Public Health 2011;39(2):156-63.

42. Satyapal KS. Ethics, transplantation, and the changing role of anatomists. Clin Anat. 2005; 18(2):150-3.

43. Prasad GVR. Understanding the sex disparity in living kidney donation. J Eval Clin Pract 2018;24(5):999-1004.

44. Quick BL, LaVoie NR, Reynolds-Tylus T, Bosch D, Morgan SE. Does Donor Status, Race, and Biological Sex Predict Organ Donor Registration Barriers. J Natl Med Assoc 2016;108(3):140-6.

45. Naderi G, Azadfar A, Yahyazadeh SR, Khatami F, Aghamir SMK. Impact of the donor-recipient gender matching on the graft survival from live donors. BMC Nephrol 2020;21(1):5.

46. Risberg G, Johansson EE, Hamberg K. A theoretical model for analysing gender bias in medicine. Int J Equity Health 2009;8:28. 
47. Hamberg K, Risberg G, Johansson EE. Male and female physicians show different patterns of gender bias: a paper-case study of management of irritable bowel syndrome. Scand J Public Health. 2004;32(2):144-52.

48. Hamberg K, Risberg G, Johansson EE, Westman G. Gender bias in physicians' management of neck pain: a study of the answers in a Swedish national examination. J Womens Health Gend Based Med. 2002;11(7):653-66.

49. Mindikoglu AL, Regev A, Seliger SL, Magder LS. Gender disparity in liver transplant waiting-list mortality: the importance of kidney function. Liver Transpl 2010;16(10):1147-57.

50. Sutton AL, He J, Edmonds MC, Sheppard VB. Medical Mistrust in Black Breast Cancer Patients: Acknowledging the Roles of the Trustor and the Trustee. J Cancer Educ 2019;34(3):600-7.

51. Cahill S, Taylor SW, Elsesser SA, Mena L, Hickson D, Mayer KH. Stigma, medical mistrust, and perceived racism may affect PrEP awareness and uptake in black compared to white gay and bisexual men in Jackson, Mississippi and Boston, Massachusetts. AIDS Care 2017;29(11):1351-8.

52. Brincks AM, Shiu-Yee K, Metsch LR, Del Rio C, Schwartz RP, Jacobs P, et al. Physician Mistrust, Medical System Mistrust, and Perceived Discrimination: Associations with HIV Care Engagement and Viral Load. AIDS Behav 2019;23(10):2859-69.

53. Jaiswal J. Whose Responsibility Is It to Dismantle Medical Mistrust? Future Directions for Researchers and Health Care Providers. Behav Med 2019;45(2):188-96.

54. Glance LG, Wissler R, Glantz C, Osler TM, Mukamel DB, Dick AW. Racial differences in the use of epidural analgesia for labor. Anesthesiology 2007;106(1):19-25.

55. Hoffman KM, Trawalter S, Axt JR, Oliver MN. Racial bias in pain assessment and treatment recommendations, and false beliefs about biological differences between blacks and whites. Proc Natl Acad Sci USA 2016;113(16):4296-301.

56. Alsan M, Wanamaker M. Tuskegee and the Health of Black Men. Q J Econ 2018;133(1):407-55.

57. Manninen DL, Evans RW. Public attitudes and behavior regarding organ donation. JAMA 1985;253(21):3111-5.

58. Bruzzone P. Religious aspects of organ transplantation. Transplant Proc 2008;40(4):1064-7.

59. Wakefield CE, Watts KJ, Homewood J, Meiser B, Siminoff LA. Attitudes toward organ donation and donor behavior: a review of the international literature. Prog Transplant 2010;20(4):380-91.

60. Hinck BD, Naelitz BD, Jackson B, Howard M, Nowacki A, Modlin CS. Assessing transplant attitudes: understanding minority men's perspectives on the multifarious barriers to organ donation. J Racial Ethnic Health Disparities 2017;4(4):580-6.

Acknowledgements: Nil.

Conflict of interest: Nil

Funding: Nil 